\title{
Abdominal Tuberculosis
}

\author{
Ismail $\mathrm{M}^{1}$, Azam $\mathrm{G}^{2}$
}

\begin{abstract}
Abdominal tuberculosis constitute up to $12 \%$ of extrapulmonary $T B$ and is sixth frequent site of extrapulmonary involvement. The most common sites of involvement is the ileocaecalregion. Other site of involvement in descending order are ascending colon jejunum, appendix, duodenum, stomach, esophagus, sigmoid colon and rectum. Abdominal TB has diagnostic dilemma due to its diverse and non-specific clinical presentation and has no single most specific, sensitive diagnostic test. A high index of suspicion, common and rare clinical feature, adequate imaging study, endoscopy, enteroscopy, laparoscopy, laparotomy, biopsy with histopathology, Mycobacterial isolation, Quantiferon-TB Gold, GeneXpert Assay, MULTIPLEX $P C R$ and clinical response to anti TB therapy are considered for early diagnosis to reduce morbidity and mortality. Six month antiTB regime is effective as nine or 12 month therapy. MDR TB and frequent interruption of therapy should considered in nonresponder to standard therapy. Surgery is required for minority cases that developed complications not responding to medical therpy.
\end{abstract}

1. Corresponding Author: Dr. Md. Ismail, MD Assistant Professor, Department of Gastroenterology Dhaka Medical College, Dhaka e-mail: drismailmd28@gmail.com

2. Dr. Golam Azam Assistant Professor, GHPD BIRDEM, Dhaka

\section{Introduction}

Tuberculosis is a infectious disease which has affected mankind since Neolithic era ${ }^{1}$. It was recognized and described by Hippocrates in his book I of the epidemics, as consumption, phthisis(waste away). Scrofula, Pott's disease, and the White Plague are other terms used to refer to tuberculosis and thought as hereditary disease. Aristotle disagreed, believing the disease was contagious. Abdominal tuberculosis usually occur in following four forms depending on the affected parts(in descending incidence) nodal, peritoneal, gastrointestinal and visceral or solid organ excluding genitourinary system ${ }^{2}$. The gastrointestinal tract from mouth to anus, peritoneum and pancreatobiliary system may be involved in abdominal TB.

\section{Epidemiology of tuberculosis}

Globally, TB incidence has fallen by an average of $1.5 \%$ per year since 2000 and is now $18 \%$ lower than the level of 2000. From 2016, the goal is to end the global TB epidemic by implementing the End TB Strategy with targets linked to the newly adopted SDGs, the strategy serves to reduce the number of TB deaths by $90 \%$ by 2030 (compared with 2015 levels), cut new cases by $80 \%$ and ensure that no family is burdened with catastrophic costs due to $\mathrm{TB}^{3}$.

Rates per100 000 population

BANGLADESH POPULATION (THOUSANDS)a 159 078, MORTALITY 51 ( 37-68), HIV-POSITIVE-TBMORTALITY 0.1 (0-0.1), PREVALENCE -404 (211659), INCIDENCE-227 (200-256), HIV PREVALENCE IN INCIDENT TB CASES (\%) $0.2(0.1-0.2)^{4}$.

Exact epidemiology of extrapulmonary TB in Bangladesh is not known. One retrospective study was done by Rouf HMA in general Hospital, Sirajgonj ${ }^{5}$ with intestinal obstruction (25\%), and $27 \%$ chronic symptoms. Faiz M.A. did another retrospective study in 1989 in IPGM \& R on extrapulmonary tuberculosis and found intestinal tuberculosis in 5 cases out of 47 patients having extrapulmonary tuberculosisa ${ }^{6 a}$. For Bangladesh, a joint reassessment of estimates of TB disease burden will be undertaken following completion of the national TB prevalence survey.

\section{Pathogenesis}

There are various ways tuberculosis can spread into the abdomen and gastrointestinal tract. One route is through ingestion of the tuberculous bacilli. The second Path is from hematogenous spread from a primary lung focus during childhood with subsequent reactivation, another mechanism is through the lymphatics from infected nodes. Lastly, tuberculosis of the gastrointestinal tract can also come from direct spread from adjacent infected structures $^{7}$. Organisms are Mycobacterium tuberculosis and Mycobacterium bovis. Primary intestinal tuberculosis is usually caused by M. bovis and results from ingestion of contaminated milk from infected cows ${ }^{8}$. The most common site of involvement is the ileocaecal region, possibly because of the increased physiological stasis, increased rate of fluid and electrolyte absorption, minimal digestive activity and an abundance of lymphoid tissue at this site. Abdominal lymph nodal and peritoneal tuberculosis may occur without gastrointestinal involvement in about one third of the cases ${ }^{9}$. Enlarged 
Lymph nodes at the root of the mesentery may cause obstruction to the third part of the duodenum. Portal hypertension due to portal vein compression and obstructive jaundice due to compression of the common bile duct due to tuberculous nodes, have been reported.

\section{Pathology}

Tuberculous granulomas are initially formed in the mucosa or the Peyer's patches and tend to beconfluent. Tubercular ulcers are relatively superficial and usually do not penetrate beyond the muscularis ${ }^{10}$ and are usually transversely oriented. Cicatrical healing of these circumferential 'girdle ulcers' results in strictures. Mesenteric lymph nodes may be enlarged, matted and may caseate. Characteristic granulomas may be seen only in the mesenteric lymph nodes ${ }^{11}$. Hoon ${ }^{11}$ et al originally classified the gross morphological appearance of the involved bowel into ulcerative, ulcerohyperplastic and hyperplastic varieties. Ulcerohypertrophic types usually seen at Colon and ileocaecal regions and presents with a right iliac fossa lump.

\section{Tuberculousperitonitis(PT)}

Typed with (1) wet type with ascites, (2) encysted (loculated) type with a localized abdominal swelling; and (3) fibrotic type with abdominal masses. Clinically PT may be acute or chronic. The peritoneum is studded with multiple yellow-white tubercles. It is thick and hyperaemic with a loss of its shiny luster. The omentum is also thickened ${ }^{12}$.

\section{Disseminated Abdominal Tuberculosis}

Involving the gastrointestinal tract, peritoneum, lymph nodes and solid viscera has also been described ${ }^{13}$. Chen et al ${ }^{14}$ reported disseminated involvement of the abdomen in 21 Out of 60 patients with large bowel tuberculosis, while most of the 96 patients with tuberculous hepatitis reported by Essopet $\mathrm{al}^{15}$ had disseminated disease. Multiple Lesions are common. Bhansali ${ }^{8}$ reported that small intestinal strictures were multiple in 71 Out of 119 patients.

\section{Hyperplastic tuberculosis}

Usually occurs in the ileocaecal region, solitary or multiple lesions in the distal ileum are sometimes seen and early involvement of regional lymph nodes which may caseate.

\section{Clinical presentation}

Abdominal tuberculosis is predominantly a disease of young adults. Two-thirds of the patients are 21-40 yr old and the sex incidence is equal, although some Indian studies have suggested a slight female predominance. The clinical presentation of abdominal tuberculosis can be acute, chronic or acute on chronic. Symptoms and signs of tuberculosis are non-specific and protean. Weight loss, Fever (low grade), chronic cough, malaise, anorexia, night sweets are features developed due to cytokines released by activated macrophages. i.e. IL-1, TNF ${ }^{\mathrm{a}}$ and not by the organism itself.
Table-I: The clinical presentation depends upon the site and type of involvement ${ }^{16}$

\section{Clinical features}

\begin{tabular}{llr}
\hline Site & Type & Clinical feature \\
\hline Small intestine & Ulcerative & Diarrhoea, malabsorption \\
& Stricturous & Obstruction \\
Large intestine & Ulcerative & Rectal bleeding \\
& Hypertrophic & Lump, obstruction \\
Peritoneal & Ascitic & Pain, distension \\
& Adhesive & Obstruction \\
Lymph nodes & - & Lump, obstruction \\
\hline
\end{tabular}

Table -II: Case series of intestinal tuberculosis $17,18,19$

\begin{tabular}{lrcc}
\hline Symptoms & Mukewar et al & Makharia et al & Khan et al \\
\hline Abdominal pain & $80.6 \%$ & $90.5 \%$ & $93 \%$ \\
Weight loss & $74.6 \%$ & $83 \%$ & $47 \%$ \\
Loss of appetite & $62.7 \%$ & $69.8 \%$ & $52 \%$ \\
Fever & $40.30 \%$ & $41.5 \%$ & $46 \%$ \\
Diarrhoea & $16.4 \%$ & $37.7 \%$ & $12 \%$ \\
Constipation & $25 \%$ & $49 \%$ & $31 \%$ \\
Bleeding Per rectum & $11.9 \%$ & $16.9 \%$ & $14 \%$ \\
\hline
\end{tabular}

Esophageal Tuberculosis:

It is rare, constituting about $0.3 \%$ of GIT TB. Additonal symptoms are dysphagia, odynophagia, pain at mid third of esophagus, near carina due to proximity to mediastinal lymph node ${ }^{20}$.

\section{Gastroduodenal Tuberculosis}

About 1\%, usually involves antral region, prepyloric area, and fundus and usually non healing ulcer or hypertrophic lesion with or without the gastric outlet obstruction ${ }^{21,22}$ or perforation.

\section{Duodenal Tuberculosis}

Most common in third part and may be intrinsic (ulcerative, hypertrophic or ulcerohypertrophic) orextrinsic (periduodenal lymph nodes compression from the outside). The largest published series of duodenal tuberculosis reported 30 cases from India ${ }^{23}$; most patients (73\%) had symptoms of duodenal obstruction (mostly extrinsic compression). The remainder (27\%) had a history of dyspepsia and were suspected of having duodenal ulcers. Two of these patients presented with hematemesis. Other reported complications by various authors are perforation ${ }^{24}$ fistulae (pyeloduodenal, duodenocutaneous, blind $)^{25}$ and obstructive jaundice by compression of the common bile duct. Recently Mohite et $\mathrm{al}^{26}$ from Mumbai reported a case of duodenal tuberculosis presenting with choledocho-duodenal fistula.

\section{Ileocaecal Tuberculosis}

Patient may present with acute tubercular abdomen(intestinal obstruction - acute or acute on chronic. Peritonitis with or without perforation, Acute mesenteric 
lymphadenitis, Acute tubercular appendicitis) ${ }^{27}$. Or As Subacute intestinal obstruction - colicky abdominal pain, distension, vomiting, gurgling, feeling of a ball of wind moving in the abdomen, and visible loops and peristalsis; thats relieved spontaneously after passage of flatus. Abdominal examination may reveal no abnormality or a doughy feeling to a well defined, firm, usually mobile mass palpablein the RLQ of the abdomen. Associated lymphadenitis is responsible for the presence of one or more lumps which are mobile if mesenteric nodes are involved and fixed if para aorticor illiac group of nodes are enlarged. Or as complication-small intestinal perforation(5-9\% all small intestinal perforation in india) ${ }^{28}$ malabsoption 29

\section{Isolated or segmented colonic tuberculosis}

It may $(9.2 \%$ of abdominal $\mathrm{TB})$ presents with pain, mild hematochezia usually involve sigmoid, ascending and transverse colon ${ }^{30}$.

\section{Rectal and Anal tuberculosis}

Haematochezia is the most common symptom (88\%) followed by constitutional symptom (75\%) and constipation $(37 \%){ }^{31}$ The high frequency of rectal bleeding may be because of mucosal trauma caused by stool traversing the strictured segment. Digital examination reveals an annular stricture. The stricture is usually tight and of variable length with focal areas of deep ulceration. ${ }^{31}$ Also presents as stricture, fistula-inano $^{32}$ or fissure-in-ano. Tubercular fistula-in-ano are multiple.

\section{Investigations}

Routine test commonly includes CBC- reveal mild anaemia and increased ESR in 50 to $80 \%$ of patients. The white blood count is usually normal, Hypoalbuminemia common. Sputum analysis can reveal associate PTB in less than $20 \%$ cases $^{4}$. Chest $\mathrm{x}$-ray found positive for active or healed lesion of $46 \%, 39 \%, 25 \%$ by Sharma et al, Prakashseries,Tendon et al in a case series of 70,300 abdominal $\mathrm{TB}^{54}$ respectively. TST positive in $53 \%{ }^{33}$. Ascitic fluid examination, X-rays and barium studies, CT scan of Abdomen with contrast, QFT-G, Gene-Xpert MTB RIF assay, PCR for tubercular peritonitis, Diagnostic Laparoscopy for abdominal tuberculosis in peritoneal seedling and extraluminal intestinal TB, Endoscopy for upper Gastrointestinal tuberculosis can be done according to site and specific area. Chest radiographs may show hilar lymphadenopathy or tuberculous lesion in case of concurrent active pulmonary TB. However a normal chest radiograph does not rule out the possibility of abdominal tuberculosis ${ }^{34}$. Ultrasound: It is useful for imaging peritoneal tuberculosis. The following features may be seen, usually in combination ${ }^{35}$. i. Free or loculated Intraabdominal fluid; Pelvic collection may be septated and mimic ovarian cyst. ii. "Club sandwich" or "sliced bread" sign. iii. Lymphadenopathy may be discrete or conglomerated (matted). The echotexture is mixed Heterogenous. Small discrete anechoic areas representing zones of caseation may be seen within the nodes. Both caseation and calcification are highly suggestive of a tubercular etiology, neither being common in malignancy related lymphadenopathy. iv. Bowel wall thickening is best appreciated in the ileocaecal region. The thickening is uniform and concentric. v. Pseudo kidney sign - involvement of the ileocaecal region which is pulled up to a subhepaticposition. Several radiographic signs (barium meal and enema) have been described for ileocecal TB :( barium meal). 1. Chicken intestine"hypersegmentation of the contrast column. 2. Hourglass stenosis"-luminal stenosis with smooth but stiff contoursin small intestine Barium Enema. 3. "Fleischner" or "inverted umbrella" sign-thickening of the valve or wide gaping of the valve with narrowing of the terminal ileum ${ }^{36} .4$. "Goose neck deformity"-loss of normal ileocecal angle and dilated terminal ileum, appearing suspended from a retracted, fibrosed caecum. 5. "Stierlin sign"-lack of barium retention in the ileum, caecum and ascending colon, with a normal configured column of barium on either side; narrowing of the terminal ileum with rapid emptying into a shortened, rigid or obliterated caecum.

6. "String sign"-persistent narrowing indicating stenosis of the terminal ileum, sometimes annular, ("napkin ring") or with dilatation of the more proximal ileal segment("purse string") for enema. Both Stierlin and String signs can also be seen in CD and hence are not specific for TB. "Conicalcaecum", due to contraction and fibrosis of mesocolon caecum reduced in size and pulled out of iliac fossa, hepatic flexure is also pulled down.

CT scan: Iliocaecal tuberculosis is usually hyperplastic and well evaluated on CT scan. In the early, slight symmetric circumferential thickening of caecum and terminal ileum. Laterileocaecal valve and adjacent medial wall of the caecum is asymmetrically thickened. In more advanced disease gross wall thickening, adherentloops, large regional nodes and mesenteric thickening can together form a soft tissue mass centered around ileocaecal junction $^{37}$. CT scan can also pick up ulceration or nodularity with in terminal ileum, along with narrowing and proximal dialtaion. Wall thicking, ulceration and narrowing of lumen may involve in other part of small and large bowel. In the colon, involvement around the hepatic flexure is common. Complication of perforation, abscess and obstruction are also common. Tubercularascitic fluid is of high attenuation value $(25-45 \mathrm{HU})$ due to high protein content. Strands of fine septa and debris with in fluid are the characterstics, but are better appreciated on USG. Thicken peritoneum and enhenching peritoneal nodules may be seen. Omental thickening is well seen as an omental cake appearance. CT showed that abdominal tuberculous lymphadenopathy involved predominately the mesenteric, upper and lower para-aortic, periportal, and pancreaticoduodenal regions. Capsule Endoscopy and Enteroscopy: There is limited data regarding capsule endoscopy in intestinal TB . Colonoscopy: Shah et $\mathrm{al}^{38}$ has described the frequency of distribution of colonic TB based on the colonoscopy as follows: $32 \%$ disease 
confined to the ileocaecal region, 28\% ileocaecal and contiguous involvement of variable lengths of the ascending colon, 26\% segmental colonic tuberculosis with involvement of the ascending colon in $10 \%$, transverse colon in $12 \%$, and descending colon in $4 \%$; $10 \%$ ileocaecal and non-confluent involvement of another part of the colon and in $2 \%$ the entire colon was affected. The Colonoscopic findings in various series in patients of GI tuberculosis are high lightened in Table III. ${ }^{39-40}$ The main differential diagnosis at endoscopy is Crohn's disease (CD). As use of steroids for a misdiagnosis of $\mathrm{CD}$ may have disastrous consequences in patients with TB enteritis. Colonoscopy with retrograde intubation of the ileum is the initial procedure of choice to differentiate. In patients with suspected or proven CD, ileocolonoscopy provided similar sensitivity $(67 \%$ vs. $83 \%)$ but significantly higher specificity $(100 \%$ vs. $53 \%)$ compared to video capsule endoscopy ${ }^{41}$. The diagnostic yield of histology increases with increasing number of biopsies from up to four segments in the colon. Endoscopic biopsies from segments upstream after dilating a stricture, and also from the normal looking ileum, increase the yield in patients with suspected TB.

Table -III : Colonoscopic findings

\begin{tabular}{|c|c|c|c|c|}
\hline & Alvareset al & Misra SP et al & SinghVet al & DasHS \\
\hline Ulceration & $70 \%$ & $92 \%$ & $83 \%$ & $47 \%$ \\
\hline Nodularity & $56 \%$ & $88 \%$ & $79 \%$ & $42 \%$ \\
\hline
\end{tabular}

Deformed

Caecum and

\begin{tabular}{|c|c|c|c|c|}
\hline IC valve & $40 \%$ & $42 \%$ & $55 \%$ & NA \\
\hline Strictures & $23 \%$ & $25 \%$ & $27 \%$ & $14 \%$ \\
\hline Polypoidlesion & ns $14 \%$ & $6 \%$ & $5 \%$ & $4.7 \%$ \\
\hline \multicolumn{5}{|l|}{ Segmental } \\
\hline Involvement & $19 \%$ & $22 \%$ & $19 \%$ & $14 \%$ \\
\hline Fibrous bands & $7 \%$ & $8 \%$ & NA & NA \\
\hline \multicolumn{5}{|c|}{ Lesions mimicking } \\
\hline Carcinoma & $16 \%$ & NA & $20 \%$ & NA \\
\hline
\end{tabular}

USG \& EUS guided FNA: Suri et $\mathrm{al}^{42}$ in his series of abdo. Lymphadenopathy found $58 \%$ positive diagnosis of abdominal tuberculosis at FNAC. Puri et $\mathrm{al}^{43}$ found $90.8 \%$ positive diagnosis(of failed USG guided FNAC) patients; $76.1 \%$ were found to have tuberculosis. Dhir et $\mathrm{al}^{43}$ studied the utility of EUS-FNA in evaluating intraabdominal lymph nodes of unknown etiology, in the setting of high endemicity of tuberculosis. Sensitivity, specificity, PPV and NPV for diagnosing tuberculosis via EUS-FNA were $97.1 \%, 100 \%, 100 \%$ and $96.9 \%$, respectively. Ascitic Fluid examination: The tubercular ascitic fluid has protein more than $3 \mathrm{~g} / \mathrm{dL}$, with a total cell count of $150-4000 / \mu \mathrm{L}$ and consists predominantly of lymphocytes. The ascitic fluid to blood glucose ratio is less than 0.96 and serum ascitic albumin gradient is less than $1.1 \mathrm{~g} / \mathrm{dL}^{44}$. The yield of organismson smear and culture is low. Staining for acid fast bacilli is positive in less than $3 \%$ of cases and a positive culture is seen in only $20 \%$ of cases. Ascitic fluid adenosine deaminase (ADA) levels are elevated in tubercular ascites. Serum ADA level above $54 \mathrm{U} / \mathrm{L}$, ascitic fluid ADA level above $36 \mathrm{U} / \mathrm{L}$ and an ascitic fluid to serum ADA ratio more than 0.98 are suggestive of tuberculosis ${ }^{45}$. However, in cases of coinfection with HIV, ascitic ADA levels can be normal or low. Also, falsely high values can be seen in malignant ascites. Interferon- $\gamma$ levels are also elevated in tubercular ascites ${ }^{46}$. The sensitivity and specificity increased by combining ascitic fluid ADA and interferon- $\gamma$ assay. Histopathology: Microbiological Diagnosis of abdominal tuberculosis is difficult; the yield of organisms from abdominal lesions is low because extra pulmonary disease is paucibacillary. Acid-fast Bacilli were seen on histological examination by Ziehl Nielson Staining in only $6-8 \%$ of patients ${ }^{47}$. The Diagnosis of abdominal tuberculosis is therefore mainly histological-Epithelioid cell granulomas with Langhan's Giant cells, peripheral rim of lymphocytes and plasma cells, and central caseation necrosis. Non-caseating granulomas, as seen in Crohn's disease, may be present in tuberculosis due to low virulence of organisms and increased host resistance. Mycobacterial Culture should be performed in all cases (although Results take 6 weeks) because it may be positive even in the absence of a characteristic histological picture ${ }^{48}$. Alvares et al in his study demonstrated wellformed granulomas in 23 patients (54\%). 14 of the patients $(61 \%)$ had caseation and $11 \quad(48 \%)$ had confluence of the granulomas. Acid-fast bacilli were present in the biopsies from two patients (5\%). Recently Ihama et $\mathrm{al}^{49}$. demonstrated the diagnosis of intestinal tuberculosis using amonoclonal antibody to Mycobacterium tuberculosis. The antibody being to the CD 68 present in the granuloma. Quantiferon-TB Gold (QFT-G) and GeneXpert Assay: The QuantiFERON-TB Gold In-Tube (QFT-G) is a blood test for use as an aid in diagnosing Mycobacterium tuberculosis infection (both latent and active tuberculosis disease). The test is approved by the U.S. Food and Drug Administration (FDA) for use with adult patients. Interferon-Gamma Release Assays (IGRAs) may be used in place of (but not in addition to) a TST in all situations in which the CDC recommends TST as an aid in diagnosing $\mathrm{M}$. tuberculosis infection. IGRA is preferred for testing persons who have received $\mathrm{BCG}$, to test recent contacts of persons with infectious tuberculosis with follow-up testing. In contact investigations, negative results obtained prior to 8 weeks typically should be confirmed by repeat testing 8-10 weeks after the end of exposure. The QFT-G is an indirect test for $M$. tuberculosis infection that is based on measurement of a cell-mediated immune response. A cocktail of 3 mycobacterial proteins (ESAT-6, CFP-10, 
and TB 7.7) stimulate the patient's T-cells in vitro to release interferon-gamma, which is then measured using ELISA technology. The test detects infections produced by the M. tuberculosis complex proteins; thus, patients either vaccinated with BCG or infected with environmental mycobacteria should test negative. Results should always be interpreted in conjunction with other clinical and laboratory findings. In a review of metaanalysis $^{50}$ the pooled sensitivity, specificity, positive likelihood ratio, and negative likelihood ratio of IGRA for the diagnosis of ITB was $81 \% \quad(95 \%$ CI, 75$86 \%), 85 \%$ (95\% CI, 81-89\%), 6.02 (95\% CI: $4.62-$ 7.83), and 0.19 (95\% CI: 0.10-0.36) The AUC was 0.92 . IGRAs do not have high accuracy for the prediction of active TB, although use of IGRAs in some populations might reduce the number of people considered for preventive treatment. Several longitudinal studies show that incidence rates of active $\mathrm{TB}$, even in IGRA-positive individuals in high TB burden countries, are low, suggesting that a vast majority ( $>95$ percent) of IGRA-positive individuals do not progress to TB disease during follow-up ${ }^{51}$. The latest guidelines from the United States, Canada,the European Centre for Disease Prevention and Control (ECDC), the United Kingdom, and World Health Organization (WHO) do not support the use of QFT-G in the setting of active TB. WHO recommendation 2013; Gene Xpert MTB/RIF may be used as a replacement test for usual practice (including conventional microscopy, culture or histopathology) for testing specific nonrespiratory specimens (lymph nodes and other tissues) from patients suspected of having extra pulmonary TB (conditional recommendation, very lowquality evidence). Gene Xpert MTB/RIF test is a simple method, and routine staff with minimal training can use the system. The test appeared to be as sensitive as culture with smear-positive specimens but less sensitive with smear-negative pulmonary and extrapulmonary specimens that include low numbers of bacilli ${ }^{52}$. MULTIPLEX PCR: Multiplex PCR using MPB64 and IS6110 are useful in rapid diagnosis of gastrointestinal TB. Multiplex PCR has sensitivity and specificity of $90 \%$ and $100 \%$, respectively in confirmed (AFB/culture/histopathology) cases of gastrointestinal TB and positive results in $72.41 \%$ of the suspectedgastrointestinal TB cases ${ }^{53}$.

Lapaorscopy: Laparoscopy provides a good deal of visual confirmation of findings, taking biopsy and collecting ascitic fluid for further investigations. Bhargava et $\mathrm{al}^{54}$ reported laparoscopic findings in 38 proven cases of peritoneal tuberculosis. The laparoscopic appearances can be classified into three types: thickened peritoneum with military yellowish white tubercles with or without adhesions ( $\mathrm{n}=25)$, only thickened peritoneum with or without adhesions $(n=8)$, and fibro adhesive pattern $(n=5)$. Biopsies were avoided from fibro adhesive lesions due to risk of complications. Visual diagnosis was accurate in $95 \%$ of patients. In comparison, in $27(82 \%)$ of 33 patients, the examination enabled a histological diagnosis to be made on the basis of typical granuloma.

\section{Diagnosis}

Timely diagnosis based on a high index of suspicion in areas and in populations in which tuberculosis is common, an algorithmic diagnostic approach using radiology, imaging and endoscopy (table-II), and management with a judicious combination of anti-tubercular therapy and conservative surgery (table- III), can reduce the mortality of this 'easily curable yet potentially lethal'disease $\mathrm{e}^{55}$.

Table IV: Diagnostic algorithm

\begin{tabular}{lcll}
\multicolumn{3}{c}{ Possible lesions } \\
\hline Presentation & \multicolumn{1}{c}{ Site } & Type & Investigations \\
\hline Intestinal Obstruction & Small Intestine & Stricturous & Small Bowel Enema \\
& Peritoneum & Adhesive & \multicolumn{1}{c}{ CT } \\
Lump & Ileocaecalreg., Colon & Hypertrophic DCBE \\
& Lymph Nodes & & Colonscopy+Biopsy \\
& Small Intestine & Ulcerative & US, CT, FNA \\
Diarrhoea & & & Small Bowel Enema, \\
& Large Intestine & Ulcerative & Colonoscopy+Biopsy \\
Rectal Bleeding & Peritoneum & & Ascitic US, CT, Ascitic tap, \\
Ascites & & & Laparoscopy+Biopsy \\
& & & + ADA. \\
\hline
\end{tabular}

Other tests:Quantiferon-TB Gold (QFT-G)(WHORecommondation)

GeneXpertAssay(replacment of conventional, microscopy, culture or histopathology)

Interferon-Gamma Release Assays (IGRAs)(Replacement of TST).

MULTIPLEX PCR (Rapid diagnosis)

Table -V: Management

\begin{tabular}{llc}
\hline Site & \multicolumn{1}{c}{ Type } & Suggested Treatment \\
\hline Any Site & Acute Abdomen & Emergency Surgery \\
Intestinal & Ulcerative & ATT \\
& Stricture & Strictureplasty, resection \\
& Hypertrophic & Resection \\
Peritoneal & Ascitic, Adhesive & ATT+Steroids \\
Lymph node & & ATT \\
\hline
\end{tabular}

Management

All Patients with abdominal tuberculosis should receive a full course of anti-tubercular therapy. Conventional Regimens include anti-tubercular therapy for 12 To 18 months. Short-course Regimens including ethambutol, rifampicin and isoniazid for 3 months followed by rifampicin and isoniazid for 6 Months or pyrazinamide, ethambutol, rifampicin and isoniazid for 2 months followed by rifampicin and isoniazidfor 4 months, are effective for abdominal tuberculosis ${ }^{56}$. It is important to administer a correct and complete course, as inadequate 
drugs, dose or duration is the most important cause of emergence of multi-drug-resistant tuberculosis. As many as $20 \%{ }^{57}$ to $40 \%$ of patients with abdominal tuberculosis who present with acute abdomen require emergency surgical intervention ${ }^{58}$. Acute-on-chronic intestinal obstruction usually responds to conservative management; these patients can then be electively investigated and managed accordingly ${ }^{59}$. Tubercular Perforations are usually ileal and are associated with distal strictures; if the two are close to each other the segment should be resected. Parikh' Described stricture plasty in such situations the incision through the stricture encircling the perforation. If they are far apart the perforation may be closed after freshening the edges and the stricture may be resected or treated with strictureplasty ${ }^{60}$. In Patients with acute tuberculous peritonitis and acute mesenteric lymphadenitis, biopsy alone is performed and the abdomen is closed without drainage."Peritoneal Toilet should be performed. Patients with ulcerative intestinal disease and those with peritoneal and lymph node involvement may be treated with anti-tubercular therapy if no complications are present. In patients with peritoneal disease, the addition of steroids may reduce the subsequent complications of adhesions. Since most patients with strictures and hypertrophic lesions have obstructions, surgical treatment is recommended.

\section{Referances}

1. Mc Dermott LJ, Glassroth J, Mehta JB, Dutt AK. Tuberculosis Part-1. Dis Mon. 1997;43(3):113-80.

2. Manohar A, Simjee AE, Haffejee AA. Symptoms and investigative findings in 145 patients with tuberculous peritonitis diagnosed by Peritoneoscopy and biopsy over a five year period. Gut.1990;31:1130-2.

3. WHO-Global tuberculosis report -2015-I,15.

4. Rouf HMA. Intestinal tuberculosis in Bangladesh study 43 cases. Journal of Bangladesh College of Physicians and Surgeons.1990;7(2):38-44.

5. Faiz MA, Das KK, Khondaker AK, Tahir M. Extra pulmonary tuberculosis in Bangladesh - A Reviewof 47 cases. Journal of Bangladesh College of Physicians and Surgeons. 1990;17(2):1-7.

6. Andronikou S, Welman CJ, Kader E. The CT features of abdominal tuberculosis in children. Pediatr Radiol.2002;32:75- 81.

7. Zissin R. Computerized Tomography Findings of Abdominal Tuberculosis: Report of 19 Cases. IMAJ.2001;3:414-418.

8. Bhansali SK. Abdominal tuberculosis. Experiences with 300 cases. Am J Gastroenterol. 1977;67:324-37.

9. Prakash A. Ulcero-constrictive tuberculosis of the bowel. Int Surg. 1978;63:23-9.

10. Hoon JR, Dockerty MB, Pemberton J. Ileocaecal tuberculosis including a comparison of this disease with non-specific regional enterocolitis and noncaseous tuberculate denterocolitis. Int Abstr Surg.1950;91:417- 40.
11. Jadvar H, Mindelzun RE, Olcott EW, Levitt DB. Still a great mimicker: abdominal tuberculosis. Am J Radiology 1997; 168:1455-60.

12. Monill-serra JM, Martinez-Noguera A, Montserrat. Abdominal ultrasound of disseminated tuberculosis in AIDS. J clin Ultrasound.1997;25:1-6.

13. Chen WS, Leu SY, Lin JK, Lin TC. Trend of large bowel tuberculosis and relation with pulmonary tuberculosis. Dis Colon Rectum. 1992;35:189-92.

14. Essop AR, Posen JA, Hodkinson JH. Segal I Tuberculous hepatitis: a clinical review of 96 cases. QJI Med.1984;212:465-77.

15. Tandon RK, Bansal R, Kapur BML. A study of malabsorption in intestinal tuberculosis: stagnant loop syndrome. AmJ Clin Nutr.1980;33:244-50.

16. Saurabh Mukewar, Shrikant Mukewar, Raghvendra Ravi. Colon Tuberculosis: Endoscopic Features and Prospective Endoscopic Follow-Up After AntiTuberculosis Treatment. Clinical and Translational Gastroenterology.2012;3:e24.

17. Makharia GK, Srivastava S, Das P. Clinical, Endoscopic, and Histological Differentiations Between Crohn's Disease and Intestinal Tuberculosis. Am J Gastroenterol.2010;105:642-651.

18. Khan R, Abid S, Jafri W. Diagnostic dilemma of abdominal tuberculosis in non-HIV patients: an ongoing challenge for physicians. World J Gastroenterol. 2006;12:6371-5.

19. Jain SS, Somani PO, Mahey RC. Esophageal tuberculosis presenting with hematemesis. World J Gastrointest Endosc.2013;5:581-583.

20. Khanna AK, Kumar K, Mishra MK, Jain AK, Gupta JP. Duodenal tuberculosis: presentation in a surgical unit. Ann Nad Acad Med Sci (India).1994;30:282-4.

21. Di Placido R, Pietroletti R, Leardi S, Simi M. Primary Gastroduodenal tuberculous infection presenting as pyloric outlet obstruction. Am J Gastroenterol.1996;91:807-8.

22. Gupta SK, Jain AK, Gupta JP. Duodenal tuberculosis. Clin Radiol.1988;39:159-61.

23. Berney T, Badaoui E, Totsch. Duodenal tuberculosis presenting as acute ulcer perforation. Am J Gastroenterol. 1998;93:1989-91.

24. Nair KV, Pai CG, Rajagopal KP. Unusual presentations of duodenal tuberculosis. Am J Gastroenterol.1991;86:756-60.

25. Mohite A, Somani P, Gambhire P. Tuberculous choledocho-duodenal fistula with extrahepatic portal vein obstruction: rare association. J Formos Med Assoc.2013;112:807-9.

26. Al-Hadeedi S, Walia HS, Al-Sayer HM. Abdominal tuberculosis. Can J Surg.1990;33:233-7.

27. Gill SS, Eggleston FC. Acute intestinal obstruction. Arch Surg.1965;91:589-91. 
28. Ranjan P, Ghoshal UC, Aggarwal R, Pandey R, Misra A, Naik S, et al. Etiological spectrum spsoradicmal absorption syndrome in Northern Indian adults at a tertiary hospital. Indian J Gastreoenterol.2004;23:94-8.

29. Chawla S, Mukerjee P, Bery K. Segmental tuberculosis of the colon: a report of ten cases. ClinRadiol.1971;22:104-9.

30. PuriAS, Vij JC, Chaudhary A. Diagnosis and outcome of isolated rectal tuberculosis. Dis Colon Rectum.1996;39:1126-9.

31. Shukla HS, Gupta SC, Singh G, Singh PA.Tubercular Fistula in ano. Br J Surg. 1988;75:38-9.

32. Alyoune M, Nadir S, Merzouk M. Tuberculous anal fistulas. Ann Gastroenterol Hepatol.1994;30:9-1.

33. Shakil AO, Korula J, Kanel GC. Diagnostic features of tuberculous peritonitis in the absence and presence of chronic liver disease: a case control study. Am J Med.1996;100: 179-85.

34. Aguado JM, Pons F, Casafont F. Tuberculous peritonitis: a study comparing cirrhotic and noncirrhotic patients. J Clin Gastroenterol.1990;12:550-4.

35. Sood R. Diagnosis of abdominal tuberculosis: Role of imaging. J Indian Academ Clinical Med. 2001;2:83.

36. Engin G. Imaging of Extrapulmonary Tuberculosis. Radiographics.2000;20:471-488.

37. S Shah, V Thomas, M Mathan. Colonoscopy findings in 50 patients of colonic tuberculosis. Gut.1992;33:347-351.

38. Alvares JF, Devarbhavi H, Makhija P. Clinical, colonoscopic, and histological profile of colonic tuberculosis in a tertiary hospital. Endoscopy.2005;37:351-6.

39. Misra SP, Misra V, Dwivedi M. Colonic tuberculosis: clinical features, endoscopic appearance and management. J Gastroenterol Hepatol.1999;14:723-9.

40. Solem CA, Loftus EV Jr, Fletcher JG. Smallbowel imaging in Crohn's disease: a prospective, blinded, 4-way comparison trial. Gastrointest Endosc.2008;68:255-266.

41. Suri R, Gupta S, Gupta SK. Ultrasound guided fine needle aspiration cytology in abdominal tuberculosis. $\mathrm{Br}$ J Radiol.1998;71:723-7.

42. Puri R, Mangla R, Eloubeidi M. Diagnostic yield of EUS-guided FNA and cytology in suspected tubercular intra-abdominal lymphadenopathy. Gastrointest Endosc. 2012;75:100510.

43. Dhir V, Mathew P, Bhandari S. Endosonographyguided fine needle aspiration cytology of intraabdominal lymph nodes with unknown primary in a tuberculosis endemic region. J Gastroenterol Hepatol.2011;26:1721-4.

44. Wilkins EG. Tuberculous peritonitis: diagnostic value of the ascitic/blood glucose ratio. Tubercle. 1984;65:47-52. [PubMed]

45. Bhargava DK, Gupta M, Nijhawan S, Dasarathy S,
Kushwaha AK. Adenosine deaminase (ADA) in peritoneal tuberculosis: diagnostic value in ascitic fluid and serum. Tubercle. 1990;71:121-126. [PubMed]

46. Sathar MA, Simjee AE, Coovadia YM, Soni PN, Moola SA, Insam B, et al. Ascitic fluid gamma interferon concentrations and adenosine deaminase activity in tuberculous peritonitis. Gut.1995;36:419-421. 23

47. Palmer KR, Patil DH, Basran S, Riordan JF, Silk DBA. Abdominal Tuberculosis in urban Britain:A common disease.Gut.1985;26:129643.

48. Vij JC, Malhotra V,Choudhary V. A Clinicopathological study of abdominal tuberculosis. Indian J Tubercul.1992;39.

49. Ihama Y, Hokama A, Hibiya K. Diagnosis of intestinal tuberculosis using a monoclonal antibody to Mycobacterium tuberculosis. World J Gastroenterol.2012;18:6974-80.213-20.

50. Ng SC, Hirai HW, Tsoi KK, Wong SH, Chan FK, Sung JJ, et al. Systematic review with meta-analysis: accuracy of interferon-gamma releasing assay and antiSaccharomyces cerevisiae antibody in differentiating intestinal tuberculosis from Crohn's disease in Asians. J GastroenterolHepatol.2014; 29:1664-70.

51. Wallis RS, Pai M, Menzies D, Doherty TM, Walzl G, Perkins MD, et al. Biomarkers and diagnostics for tuberculosis: progress, needs, and translation into practice. Lancet.2010;375:1920.

52. Arzu N. Zeka1, Sezai Tasbakan2, Cengiz Cavusoglu3. Evaluation of the GeneXpert MTB/RIF Assay for Rapid Diagnosis of Tuberculosis and Detection of Rifampin Resistance in Pulmonary and Extrapulmonary Specimens. J. Clin. Microbiol. 2011 December;49(12) 4138-4141.

53. Sharma K, Sinha SK, Sharma A, Nada R, Prasad KK, Goyal K, et al. Multiplex PCR for rapid diagnosis of gastrointestinal tuberculosis. J Glob Infect Dis.2013;5:49-53.

54. Bhargava DK, Shriniwas, Chopra P. Peritoneal tuberculosis: laparoscopic patterns and its diagnostic accuracy. Am J Gastroenterol.1992;87:109-12. 115

55. Lingenfelser T, Zak J, Marks IN, Steyn E, Halkett J, Price SK. Abdominal tuberculosis: still a potentially lethal disease. Am Y Gastroenterol.1993;88:74-8.

56. Balasubramanian R, Ramachandran R, Joseph P. Interim Results of a clinical study of abdominal tuberculosis. Indian J Tubercul.1989;36:117-21.4-50.

57.Haddad FS, Ghossain A, Sawaya E, Nelson AR. Abdominal tuberculosis. Dis Colon Rectum.1987;30:724-35.

58. Kapoor VK, Gupta S, Sikora SS, Chattopad- Hyay TK, Sharma LK. Acute Tubercular abdomen. Indian J Surg.1991;53:71-5.

59. Innes JA. Non-respiratory tuberculosis. J R Coil Physicians Lond.1981;15:227-31.

60. Kapoor VK, Kriplani AK, Chattopadhyay TK, Sharma LK. Tuberculous Perforations of the small intestine. 